\section{MS20-P6 Quasiparticle band structure, spectral weights and degree of d-electron localization in Iron Silicides}

\author{
Igor S. Sandalov ${ }^{1,2}$, Natalia Zamkova ${ }^{2,3}$, Vyacheslav Zhandun ${ }^{2}$, \\ Sergei G. Ovchinnikov ${ }^{2,3}$
}

1. KTH Royal Institute of Technology, SE 10044 Stockholm, Sweden

2. L.V.Kirensky Institute of physics, Siberian Branch of Russian Academy Sciences, 660036 Krasnoyarsk, Russia

3. Siberian Federal University, 660041 Krasnoyarsk, Russia

email: sandalov@kth.se

The degree of quasiparticle (QP) localization as well as QP spectral weights (SWs) determine the cohesive energy and, hence, the degree of stability of a crystal under pressure. Both QP band structure and their SWs can be measured by angle-resolved photoemission spactroscopy (ARPES), SWs also manifest themselves in Compton-scattering experiments and in the effective mass of QPs at the Fermi surface.We report results of our ab initio calculations of the QP spectra and SWs quasiparticle for $\mathrm{Fe}_{3} \mathrm{Si}$ and $\alpha-\mathrm{FeSi} i_{2}$, performed within Hedin's GW approximation (GWA) by means of Vienna $\mathrm{Ab}$ initio Simulation Package (VASP). Comparison of the GGA-to-DFT and GWA band structures shows that both theories reflect peculiarities of the crystal structures in similarshape of the bands in certain $k$-directions, however, i) Fermi energies for GGR bands are lower by $0.5 \mathrm{eV}$ than the one for GWA for both compounds; ii) GWA density of electron states smoother than the GGR one and existing peaks in the density of QP states are shifted with respect to each other; iii) while the spectral weights of QPs for GGA calculation are equal to unity by definition, the GWA ones are decreased almost by half in both compounds and show non-monotonic behavior (see figure) only in those parts of bands where the delocalized states contribute to their formation. Both methods give $d$-electrons localized and large magnetic moment in $\mathrm{Fe}_{3} \mathrm{Si}$ of iron ion is surrounded iron ions, whereas the $S i$-atom nearest neighbors lead to delocalization of $d$-electrons and quenched magnetic moments. The $\alpha-F e S i$, where all iron ions have the $S i$ ions in nearest neighbors, is Pauli paramagnet. Estimation of diagrams responsible for the screening of Coulomb interaction within simplified model shows that GW approximation, even with inclusion of the standard vertex corrections, cannot describe the contribution from quasilocalized electrons to the spectral weights and, correspondingly, to the correlation mechanism of $\mathrm{d}(\mathrm{f})$-electron delocalization, considered earlier within the periodical Anderson model (Phys. Rev. B 62, 16370 (2000-II)). Thus, the ab initio methods for systems with strongly correlated electrons are needed where different approximations for $d(f)$ - and delocalized electrons will be used from the very beginning. The ARPES measurements for these compounds would be of great help for further understanding of underlying physics.
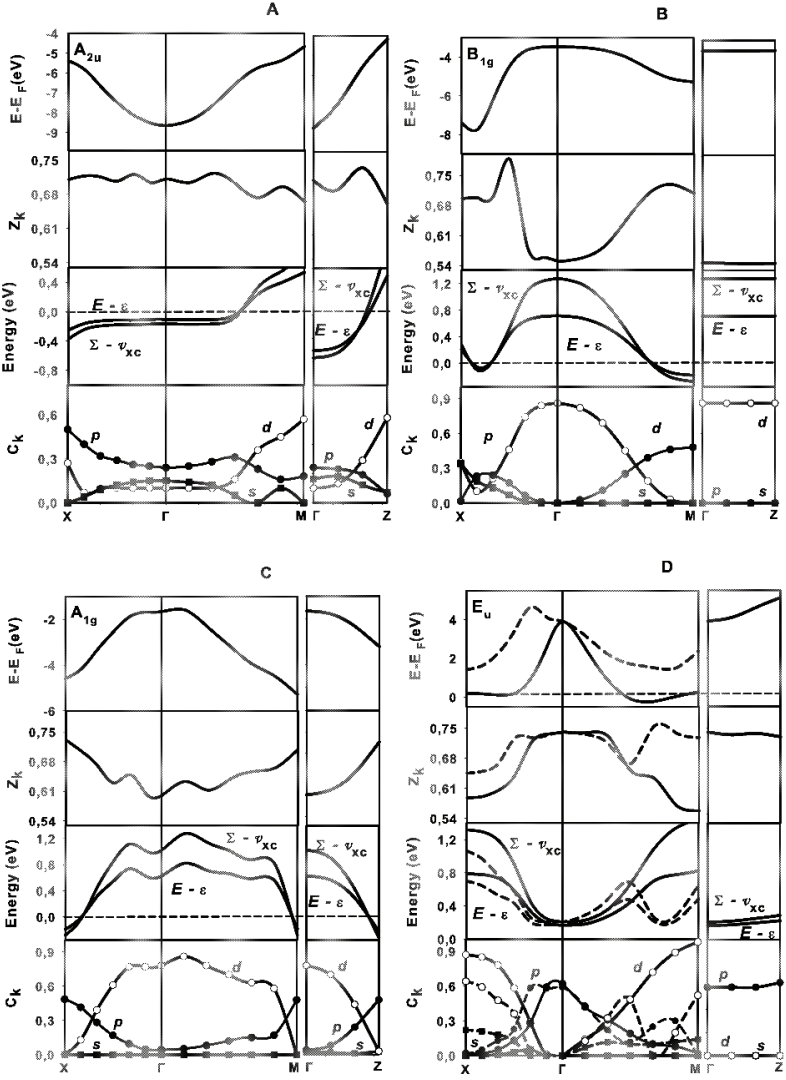

Figure 1. The $\alpha-F e S i$ bands. QP filled bands, spectral weights $Z$, the $k$-dispersion of numerator and denominator of Eq.(4) in arXiv: $1501.05898 \mathrm{v} 3$ [cond-mat.mtrl-sci] and the character coefficients $C$; d- and p- characters are denoted by empty and filled circles, the black squares stand for $s$ character.

Keywords: strongly correlated electrons, delocalization 